\title{
The Analysis of Mobile Internet Impacts on Electronic Commerce System Construction
}

\author{
Zhong $\mathrm{Wei}^{1, \text { a }}$ \\ ${ }^{1}$ Beijing University of Posts and Telecommunications, Beijing 100876, PRC \\ azhongwei1@chinaunicom.cn
}

Keywords: Mobile Internet, E-commerce system, System Construction, Open Platform

\begin{abstract}
This article analyzes the new changes of e-commerce's means and business model based on the development of mobile internet. Furthermore, Combined with the influence of the technological progress, such as wireless broadband network, mobile intelligent terminal and cloud computing, this paper puts forward an open system architecture of electronic commerce and its six module systems, and expounds the system design and deployment method in mobile internet environment.
\end{abstract}

\section{Introduction}

With the rapid development of 3G and the approaching LTE (Long Term Evolution) network revolution, the mobile internet is profoundly changing our society, economy and the whole business system. The combination of mobile communication and electronic commerce (e-commerce) led the early mobile e-commerce. The traditional mobile e-commerce is a branch and component of e-commerce, which only refers to the portion commerce activities that are associated with the mobile terminals, such as mobile payment, mobile advertising and so on. Today, a series of new techniques and applications are widely used in mobile internet industry, such as the popularity of intelligent terminals, the speed promotion of wireless broadband network, the scale application of cloud computing, which promote the integration between mobile internet and e-commerce. What is more, the connotation and scopes of e-commerce are changing deeply. These changes not only occur in mobile e-commerce domain, but also make e-commerce and mobile internet become more closely associated with each other in the future. Therefore, this paper will analyze the influence on e-commerce system construction from the development of mobile internet, so as to provide some references for building e-commerce system which is based on mobile internet.

\section{Mobile Internet Brought New Changes for Electronic Commerce}

2.1. Electronic Commerce. E-commerce means: two parties use diverse applications to complete a series of business activities by electronic equipment in open network environment. E-commerce utilizes computer technology and network communication technology, to realize the whole business process. Digitization and networking is a fundamental characteristic of e-commerce. Moreover, information flow, capital flow and logistics are three basic elements of the e-commerce activity.

According to the business model of e-commerce, it can be divided into B2B, B2C, C2C, B2G etc. Considering the long history and high turnover of $\mathrm{B} 2 \mathrm{~B}$ and $\mathrm{B} 2 \mathrm{C}$ model, they are regarded as the mainstream of e-commerce. Digital network provides realistic, reliable bases for shortening the intermediate links between production and consumption in e-commerce activities, so it is possible that the cost of e-commerce transaction will be reduced and personalized products can be provided by manufacturers rapidly. Therefore, e-commerce has brought continuous innovation.

2.2. The Development of Mobile Internet Market. By the end of 2012 April, the permeability of global 3G users is nearly $20 \%$ in the mobile subscribers, China is about $15 \%$, and the commercial LTE networks have been offered in 40 countries and regions. At present, the wireless mobile communication technology, the user identification and positioning technology, product technology, 
and mobile payment become more mature in e-commerce activities. Along with the improvement of wireless network bandwidth capability, the expansion of mobile internet users scale, the improvement of customer recognition, the acceleration of new mobile internet applications, there are obvious changes in e-commerce service field. The mobile internet becomes another impetus for e-commerce, and brings its unique advantages, such as convenience, identify ability, privacy, positioning and so on. Consequently, these advantages have brought new development spaces and new business models to e-commerce, and it will be more prominent in the future.

2.3. The New Changes of E-commerce Transaction. Traditional e-commerce platforms, such as Dangdang, Taobao, Ebay, are actively expanding into mobile field, while the desktop e-commerce is increasing mobility and continuously penetrating into the mobile terminal. Due to the mutual promotion of e-commerce and mobile internet, new changes have appeared. In general, it mainly reflects in the following three aspects: trade users, means and tools, and transaction mode.

Trade users. E-commerce user growth is restricted by the popularity of the internet, the level of consumer behavior patterns and goods distribution ability, namely the basic market conditions. Mobile internet increased the number of e-commerce users and changed their trading behaviors. The biggest characteristic of mobile internet is convenience, indicating no matter the user is in the full time or spare time, no matter at home or outside, no matter in work or in waiting, they will be able to participate in all aspects of e-commerce. It lets e-commerce possess the " $3 \mathrm{~A}$ " characteristic of mobile internet, namely, anytime, anywhere and any service. The number of e-business users grows rapidly. Although total scale of the internet users rise relatively steady, mobile users' number still maintains a high growth rate. According to CNNIC 2011 annual statistical report, mobile internet users reached 356 million in China, maintained a $17.5 \%$ growth rate. For e-commerce, It indicates that a huge number of users promote applications including online shopping, online payment, online banking, travel reservations. Various e-commerce applications grow rapidly. At the end of 2011, the number of online shopping users attained 194 million, and increased by 20.8\%. What's more, the users of the online payment and online banking increased by $21.6 \%$ and $19.2 \%$ respectively, and users were more than 160 million.

Mobile internet makes transaction process become real time, and impacts on consumer behavior. Users pay closely attention to each link of transactions. For example in the online shopping, the user not only takes part in order link, but also accesses to logistics information, payment, delivery, evaluation and sharing link through the mobile terminal and the wireless network.

Means and tools. Revolutionary development of smartphone makes mobile internet applications more feasible. The traditional e-commerce platform has penetrated into mobile terminal. Mobile internet is included in the strategy of large-scale e-commerce enterprises, and numerous mobile e-commerce applications were launched. Traditional mobile phones are less used for e-commerce due to their small screens, low processing power and simple functions. When smartphone is developed, namely many of the original computer functions are introduced to the mobile terminal, it is no longer a simple communication tool. So the features of consuming electronics and computer are emphasized. Smartphone and PAD terminal break through the bottleneck of e-commerce development. The traditional e-commerce is migrating to the mobile screen, so as to utilize the terminal mobility and the personalized information based on transactions location. It popularizes mobile payment directly, and the users are more and more dependent on the e-commerce.

Transaction mode. Mobile internet promotes the developments of e-commerce O2O (Online to Offline), B2B2C mode etc. In O2O mode, both purchase and payment are conducted online, and the user can go to the store or service provider to claim his/her purchase with electronic credentials. Users can come conveniently, quickly into the e-commerce platform in the offline entity store environment, and grasp their own transaction information or perform the second trade. Convenience, easy-location and other characteristics of mobile internet, can not only make up for the "gap" between Offline and Online, but also develop their respective advantages.

\section{Impact of Mobile Internet on Electronic Commerce Framework}


3.1. Technological Evolution Brought by Mobile Internet. Wireless broadband networks, intelligent terminals, cloud computing are three milestone progress of mobile internet era, which prominently distinguish the new mobile internet from the traditional. With the development of 3G's scale and the deployment of LTE networks, mobile communication network with a high bandwidth and low latency can provide the mobile users a better user experience and greater operational capacity. WCDMA and HSPA+ technology can achieve download speeds of 14.4Mbps, while LTE technology can provide a peak rate of downstream at 326Mbps and an upstream at $86 \mathrm{Mbps}$ in $20 \mathrm{MHz}$ spectrum bandwidth, which effectively improves the network performance, enhances residential capacity and lowers the system latency. In this case, mobile terminals can support video calls, high-definition television, online games and other wireless broadband services. Users will obtain a desktop internet experience on a mobile terminal, which creates a good network environment for e-commerce's migration to mobile terminals.

Cloud computing is an information processing method for large-scale computing through unified organizing and flexibly calling various ICT resources. Cloud computing technology utilizes distributed computing and virtual resource management through gathering the decentralized ICT resources (including computing and storage, operation platform, software, etc.) together to form a pool of shared resources, and provides services to users in dynamic on-demand and measurable ways. Cloud computing will transfer the applied computing from terminal-side to server-side. Complex calculations will be processed by the cloud, namely server-side, thereby it weakens the processing requirements on mobile terminal equipment. In this way, terminals get to take the main responsibility for the interaction with users. For reasons such as open interfaces, distributed computing concept, super and flexible processing capabilities, new types of mobile internet business based on cloud computing are being accepted by people. Mobile intelligent terminal is a functional mobile device installed with an open operating system, and can flexibly install applications, represented by smart phones, tablet PCs and e-readers. Based on the operating system, different from the traditional phones, in addition to have communication function, smartphones can also have internet access, multi-tasking, multimedia functionality, simple application installing/uninstalling and other features. The core of mobile intelligent terminal is hardware processing power, intelligent open operating system and flexible, rich mobile applications, while the importance of the communication capability is gradually reduced. Computing power has become the determinant of chip technology's development. Currently popular operating systems include Apple iOS, Google Android, and Microsoft Windows Phone. Rapid development of global smartphone market is the core driving force to promote the mobile internet. With the development of cloud computing, cloud-based intelligent terminals will emerge in large numbers, which will further reduce the cost, and enhance the deployment speed of mobile internet applications including e-commencer.

3.2. The System Framework of Open E-commerce Platform Based on Mobile Internet. On one hand, mobile internet is changing the transactions and business model of e-commerce. On the other hand, the "Cloud, Chanel, Terminal" model brought in by mobile internet technology is also gradually changing the system framework of e-commerce.

E-commerce is a digitalized and network trading activity based on open internet, which forces the system to achieve high security, high reliability, high stability, advanced scalability and openness. Safety, reliability and stability are requisite elements for e-commerce to be a business activity. As a rapidly developing application field of internet technology, it must have the characteristic of openness and scalability. Meanwhile, maintaining the advancement of the system through innovations is the key to its strong vitality, so that it can be accepted by the users and the market.

In order to adapt the development of mobile internet and the innovation of business model in the future, e-commerce system framework must have favorable openness. The e-commerce platform system should be designed in the open capacity platform. E-commerce open platform provides a range of basic services, and opens up its own interfaces. The third-party merchants can develop new business applications by applying and assembling these interfaces, and these new applications can be running on the platform uniformly. Such a service model is called e-commerce open platform, which is based on open network. E-commerce open platform system framework mainly includes six parts 
(as shown in Fig.1): platform operating system, user management system, business application system, access system, payment system and security system. Each subsystem ensures the effectiveness of the system through standardized, extensible open interfaces.

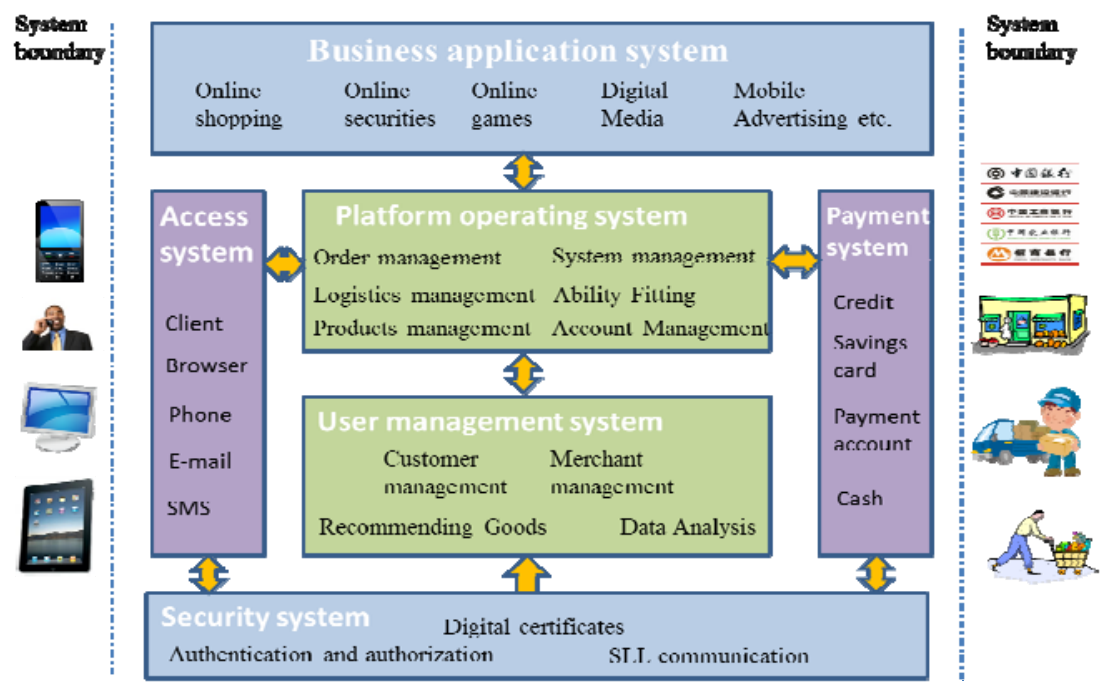

Fig. 1 E-commerce open system architecture

Business application subsystem. Business application module is mainly responsible for managing a variety of electronic transaction services. It provides a series of online service applications and application content management, such as electronic ticketing, shopping, and other applications. It is the "trading market" of e-commerce system.

User management subsystem. User management module is responsible for customers and merchants' account management. In addition to that, it provides functions such as customer identity management, opportunity recommendation and so on through consumer behavior analysis.

Platform operating subsystem. It is mainly responsible for a variety of system functions, such as website, client management, order management, billing and settlement, query statistics, and system management. It manages the open capacity as well, which includes general management and ability adaption. Common management module provides application, capacity and configuration. Capacity adaptation module provides the configuration and management of platform applications, system access, pay access, security authentication and other interface capacity to third-party applications.

Accessing Management Subsystem. Telephone voice, SMS, client, website, e-mail and other access methods for consumers are important parts of the services provided by e-commerce systems. The mobile internet has made this subsystem greatly enhanced.

Payment subsystem. It is responsible for merging the banking system, e-commerce platform system and merchants' business system, and creates unified payment rules among customers, merchants and banks. The module provides a unified model of interface for data exchanging, so a variety of payment activities can be completed by the platform based on the standardized API and interface protocol. The payment system usually supports credit card, debit card, payment account etc.

Security subsystem. The e-commerce system is a credible trading system for the enterprises and the publics through the internet. The security and reliability of the system is built on the CA safety certification mechanism, encrypted transmission and signature authentication method, so it ensures the security, integrity, consistency and accuracy of the system transaction among trading partners, customers and banks.

From the functional and administrative point of view, the e-commerce system framework is divided into six modules, where each module is independently designed, but information flows to one another by open standardized interfaces, which are also supplemented by security mechanisms. It ensures the system to have a clear structure and run stably, and it is conducive to the openness of the system's capacity for merchants. The open system framework that suits for mobile internet can 
achieve the combination of unified e-commerce platform and personalized business applications, so as to meet the innovation of e-commerce model under mobile internet.

\subsection{The Influence of Mobile Internet on the Design and Implement of E-commerce System.} The main task of the e-commerce system design is to reasonably choose software and hardware resources according to technical, economic, internal and external environment of the system, which should follow the goals of establishing an open structure. An e-commerce system generally contains a variety of business sub-systems involving numerous aspects of sales, logistics, customer service, resource management, production management etc. In terms of the open e-commerce system structure of the mobile internet, Java, due to its characteristics on the cross-platform and open-source, has been widely used. New application framework and deployment based on the J2EE technique, has been high-speedily developed at the present. The middle layers of the J2EE system structure can meet the wide range of application requirements and have high reliability, scalability and availability, and it makes the establishment and application deployment of the e-commerce system more simple and flexible. The e-commerce system design is divided into different layers including physical layer, data layer, application layer and presentation layer separately (as shown in Fig.2). The physical layer, which is the physical basis platform of the whole system, provides infrastructures for the entire system. It has different functional parts such as system resource, storage resource, networking resource and database resource. The data layer is the foundation of the system, which realizes unified organization, centralized management and shared resource of overall system data. The application layer manages all service processes, such as data calling, business logic processing, packaging, dynamic loading/unloading based on the different activation conditions. The presentation layer is a channel to communicate with the outside system, mainly responses to the operation of front desk customers and background managers, and provides appropriate information manifestation.

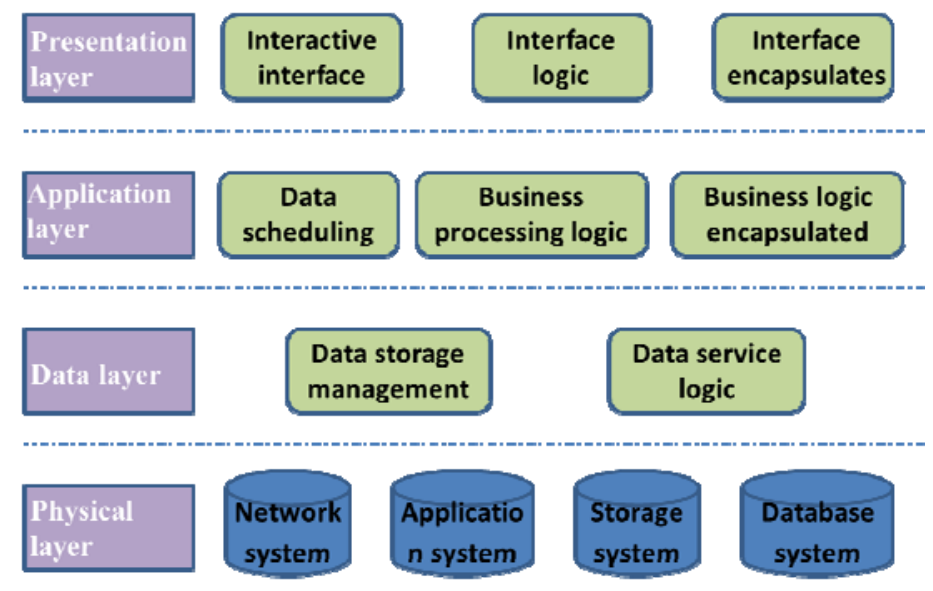

Fig. 2 E-commerce system hierarchy

With the improvement of the capacity of smart phones and other mobile intelligent terminals, a variety of client applications have developed rapidly and have been widely used in e-commerce activities. Meanwhile, access equipment and ways of e-commerce users have been changed and mobile computing has been widely used. Mobile terminals cannot always maintain steady networking connection in the presences of different networks, and wireless network environment also can cause the low reliability. Mobile computing, which is different from traditional distributed computing, has diversity and discontinuity on the network conditions. By mobile computing, useful information can be accurately and timely provided to any customers in any time and any place, which dramatically changes people's life styles and work ways. The characteristics of mobile terminals' algorithm require more complex design of e-commerce systems. According to the limitations on computation capacity and storage space of mobile terminals, the J2ME technique, which is a Java platform specially developed for small resource-constrained terminals, is applied for visiting J2EE platform in traditional e-commerce systems. On the transport layer, J2ME supports TCP/IP protocol 
and HTTP protocol. On the application layer, J2ME provides development environment for Java and enables the access of all online data by using Client/Server, which is irrelevant with markup language. However, the most recent e-commerce development is no longer constrained by aforementioned issues since the bottlenecks of its development are tackled, which owe to the improvements of transit speed of network, the developments of hardware capacity, operating system of mobile phone, and occurrence of other new techniques such as HML5.

Within the patterns of traditional e-commerce system, different business systems are operated on several mutually connected physical platforms, which provide services by internet. The traditional development patterns are not efficient for several reasons. First, resources cannot be dynamically redistributed. Second, updating the system requires rearrangement of all system resources. Hence, it is difficult to meet new requirements from the market. Moreover, maintenance of such a system is rather costly because business systems are individual and resources cannot be exchanged among them. However, cloud computing technology changes the game. Comparing to the old technology, it has advantages in virtualization, structure, distribution, and automation. These advanced features grant e-commerce more flexible and extendable through the shared resource pool. Therefore, e-commerce users can serve their clients more quickly, their resources are more dynamical, feasible, and the e-commerce system data are safer and more reliable.

\section{Conclusions}

New technology and cross-border integration have spawned a variety of new business models. The development of mobile internet has brought the complete integration of traditional desktop internet and the mobile internet, which has made the e-commerce that was born in the traditional internet era possess mobile properties increasingly. Just mobile internet makes e-commerce system can deal with trades anytime, anywhere. With these essential characteristics, the mobile e-commerce has broken through the original boundary. Promoted by mobile communication networks, intelligent terminals, cloud computing and new internet technologies, e-commerce is integrated into mobile internet. The future needs of e-commerce users and the business model will continue to undergo new changes, and it will promote the e-commerce system framework and system design to continuously develop.

\section{References}

[1] Du jia zhao. The key technologies and methods of constructing personalized smart mobile e-commerce system [D]. Lanzhou University, 2011.

[2] Sun lingfang. The construction of cloud-based e-commerce model[J].Value Engineering 2011 (6).

[3] Han xijun. Private e-commerce system building based cloud[J].Jiangsu supplier theory. 2011 (8).

[4] Jin yongqi. Internet e-commerce system and its information security[D]. Beijing University of Posts and Telecommunications, 2010.

[5] Hou shunya. MVC pattern-based e-commerce system design [D]. Dongbei University of Finance and Economics 2010.

[6] Ngai EWT, Gunasekaran A. A review for mobile commerce research and applications (J) .Decision Support Systems, 2007Volume43:(1).PP.3-15.

[7] Hou Zhixue. The realization of mobile e-commerce system based on J2ME-J2EE platform[J]. Beihua Institute of Aerospace Engineering. 2011 (5). 